\title{
Identification of Lettuce (Lactuca sativa L.) Germplasm with Genetic Resistance to Drop Caused by Sclerotinia minor
}

\author{
Rebecca Grube ${ }^{1}$ and Edward Ryder ${ }^{2}$ \\ U.S. Department of Agriculture, Agricultural Research Service, 1636 East Alisal Street, Salinas, CA 93905
}

\begin{abstract}
ADDITIONAL INDEX wORDS. Lactuca sativa, breeding, soilborne fungi, resistance evaluation, inoculation, partial
ABstract. Incidence of the disease lettuce drop caused by Sclerotinia minor is often high in California lettuce fields despite the use of cultural and chemical controls. Development of resistant lettuce cultivars has been hindered by the difficulty of evaluating resistance in field tests and the lack of a screening procedure that reliably predicts field performance. Several lettuce genotypes of diverse geographic origin and plant architecture including modern and heirloom cultivars, plant introduction accessions, and breeding lines, were evaluated for resistance to $S$. minor using several methods. Resistance was evaluated in fields that contained naturally occurring $S$. minor, in a field that contained both naturally occurring and manually incorporated $S$. minor inoculum, and in the greenhouse using two types of inocula. Many genotypes exhibited partial resistance to $S$. minor, with significantly reduced disease incidence relative to susceptible controls. The similarity of disease ratings observed in replicated field tests supports the conclusion that partial resistance is under genetic control. Ratings obtained in some greenhouse tests were highly correlated with field ratings, but this was not true for all tests. Therefore, although greenhouse evaluation with adequate replication and repetition can be used as a selection tool, field testing remains an essential component of $S$. minor resistance breeding programs.
\end{abstract}

Lettuce drop is caused by two species of Sclerotinia, S. minor Jagger and S. sclerotiorum (Lib.) de Bary. Both species produce sclerotia, persistent soilborne resting bodies that germinate as hyphae or as ascospore-producing apothecia. Sclerotinia minor infects lettuce almost exclusively by soilborne mycelia, and does not produce the infective airborne ascospores typical of $S$. sclerotiorum (Subbarao, 1998). The epidemiology and symptoms of lettuce drop caused by the two species differ markedly, and the two species are often geographically and temporally separated (Subbarao, 1998). Our study focused on S. minor, which predominates in the cooler coastal valleys of California, a major U.S. lettuce growing region (Subbarao, 2000). Sclerotinia minor has a broad host range, infecting other crops (e.g. peanut), several weed species and species used as cover crops (Hollowell et al., 2003; Koike et al., 1996).

Cultural controls including various tilling, irrigation and crop rotation strategies have been evaluated, and some have proven effective at reducing disease incidence and inoculum (Hao et al., 2003; Subbarao et al., 1996; Subbarao et al., 1997). Currently, a combination of fungicide applications and good cultural practices reduces crop losses but is expensive and does not give satisfactory control (Bardin and Huang, 2001; Subbarao, 1998). A high level of genetic resistance in crop cultivars could provide inexpensive and reliable protection.

Four types of lettuce cultivars are grown on a large scale in the U.S.: iceberg, romaine, butterhead and leaf (Ryder 1999). Other types now grown on a smaller scale or in other parts of the world include batavia, which form loose heads, stem lettuce, which is grown for its large succulent main stem, and oilseed lettuce, which

Received for publication 28 July 2003. Accepted for publication 17 July 2003. We thank C.R. Barr, S. Benzen, R. Bhat, J. Epting, J. Hubbard, A. Ibrahim, D. Milligan, D. Renteria, B. Robinson, K. Subbarao, J. Tanaka, and B.M. Wu for materials, technical assistance, and valuable discussions. We also thank L. Kohn, K. Subbarao, G. Abawi, M. Melzer, and R. Aburomia for critical review of tis manuscript. We acknowledge the California Lettuce Research Board for financial support of this work.

${ }^{1}$ Research plant geneticist. Corresponding author; e-mail rgrube@pw.ars.usda.gov. ${ }^{2}$ Collaborator. often has a weedy growth habit with extensive lateral branching. Together, iceberg and romaine types comprise $90 \%$ of all lettuce grown in the U.S., and crop losses due to S. minor are common in all commercially available cultivars of both types.

Despite extensive screening efforts, no lettuce genotype with complete resistance to lettuce drop has been identified. At least two modern cultivars have been described by their developers as being less vulnerable to attack by Sclerotinia than other cultivars, although data supporting these claims have not been documented (Seminis Vegetable Seeds, 1999; M. Massoudi, personal communication). Partial resistance has been described among wild Lactuca species, PI accessions, breeding lines, and heirloom cultivars, using either S. sclerotiorum (Chupp and Sherf, 1960; Elia and Piglionica, 1964; Madjid et al., 1983; Newton and Sequiera, 1972) or S. minor (Abawi et al., 1980; Subbarao, 1998). It is unknown whether resistance to one species is effective against the other. In most cases, partial resistance has been defined as a reduction in disease incidence (DI) and death, although Subbarao (1998) has also described slow-dying resistance, which is characterized by delayed plant death after the first appearance of symptoms, as compared with susceptible controls. In this report, we use the term partial resistance to refer to a reduction in DI as compared with the susceptible control genotype. This term is not meant to imply knowledge of the underlying mechanism of resistance, which could include physiological or tissue-based resistance, avoidance or escape, or a combination of mechanisms.

The identification and use of resistance to lettuce drop in breeding programs has been hindered by the lack of an artificial inoculation procedure that accurately predicts performance in fields infested with Sclerotinia species. All previous reports of resistance to $S$. minor in lettuce have been based on greenhouse evaluation methods, where plants were inoculated with rye seeds colonized with mycelia, sclerotia or a combination of the two (Abawietal., 1980; Subbarao, 1998). Comparisons between results obtained using these methods and field tests have not been made for $S$. minor. When evaluation methods were compared for $S$. sclerotiorum, measurements of resistance obtained in greenhouse and field tests were poorly correlated in one case (Newton and 
Sequiera, 1972) and showed limited correspondence in the other (Whipps et al., 2002).

To facilitate breeding and genetic analyses for resistance to $S$. minor in lettuce, our objectives in this study were to (i) identify lettuce genotypes that show resistance to $S$. minor in infested fields, (ii) to examine the consistency of field evaluations conducted in multiple years, and (iii) to compare two methods for evaluating resistance in the greenhouse and determine whether either method gave results that were consistently correlated with partial resistance in field tests.

\section{Materials and Methods}

Plant material. For all experiments, seeds were obtained from the USDA-ARS lettuce germplasm collection in Salinas, Calif. Plant materials are listed by experiment in which they were tested and by horticultural type (Table 1). In each experiment, the heirloom 'Batavia Reine des Glaces' ('BRG') was used as the susceptible control and 'Salinas' was used as a representative modern iceberg lettuce cultivar.

In two experiments, 1980a and 1980b, 12 head lettuce genotypes were evaluated in commercial fields. These were chosen based on their apparent resistance to $S$. minor in field trials and on prior studies of resistance to $S$. sclerotiorum in lettuce. The 12 genotypes included 'Salinas', nine heirloom cultivars and two USDA breeding lines (74-1076 and 74-1077).

Extensive greenhouse screening was performed as described to identify lettuce genotypes with higher levels of resistance to S. minor (Abawi et al., 1980; Subbarao, 1998; K. Subbarao, E. Ryder, and R. Grube, unpublished data). In 2001, 29 genotypes, including those identified as partially resistant in greenhouse experiments, were evaluated in a field infested with S. minor. Two modern commercial cultivars described as being less vulnerable to attack by Sclerotinia spp., 'Sharp Shooter' iceberg and 'Green Forest' romaine, were also included.

Twelve genotypes of varying horticultural type were evaluated in seven greenhouse and two field $(2000,2001)$ experiments to evaluate the consistency of field tests from year to year and to compare greenhouse and field evaluation procedures. These genotypes were chosen to maximize the observed variation in susceptibility to $S$. minor, based on performance in preliminary experiments.

Fungal CUlTuRE. Sclerotinia minor isolates taken from lettuce in the Salinas Valley showed different levels of virulence on lettuce, but there was no evidence for interactions between pathogen isolates and host cultivars (K. Subbarao, personal communication). A single highly virulent isolate that was isolated from a lettuce field in Santa Maria, Calif. in 1993, Sm18, was used for all experiments. The isolate was maintained as sclerotia and was routinely subcultured on potato dextrose agar (PDA) before use.

To produce rye seed inoculum, rye seeds were mixed with water $(1: 1, \mathrm{v} / \mathrm{v})$, autoclaved twice for $20 \mathrm{~min}$ and inoculated with mycelial plugs taken from the growing margin of 2-day-old PDA cultures. Cultures were incubated on laboratory benches under ambient room temperature and lighting conditions or were maintained in a growth chamber at $20^{\circ} \mathrm{C}$ with a $14 \mathrm{~h}$ photoperiod and light intensity of $90 \mathrm{~W} / \mathrm{m}^{2}$. Rye seed inoculum comprised 1 to 3 infested rye seeds taken from 21-d-old cultures.

To produce PDA plug inoculum, sclerotia were produced on rye seed as described above. Sclerotia were harvested from 21day-old cultures, rinsed with distilled water, dried for one week at room temperature and stored at $4{ }^{\circ} \mathrm{C}$ until use. Before use, sclerotia were plated on PDA and incubated $2 \mathrm{~d}$ in a growth chamber at $20{ }^{\circ} \mathrm{C}$ with a $14 \mathrm{~h}$ photoperiod. Plugs $\left(1 \mathrm{~cm}^{2}\right)$ containing mycelia from a single germinated sclerotium were excised from the PDA plate and used as inoculum.

RESISTANCE EVALUATION-FIELD. Experiments were conducted at three field sites (Table 2). Preliminary experiments (1980a and 1980 b) were conducted in two field sites in a commercial ranch in the Blanco district of the Salinas Valley. In these sites, cultivars were exposed to field populations of $S$. minor, which were not supplemented with additional inoculum. Subsequent experiments were conducted in 2000 and 2001 in a single field located at the

Table 1. Plant material evaluated for resistance to Sclerotinia minor in greenhouse and field tests.

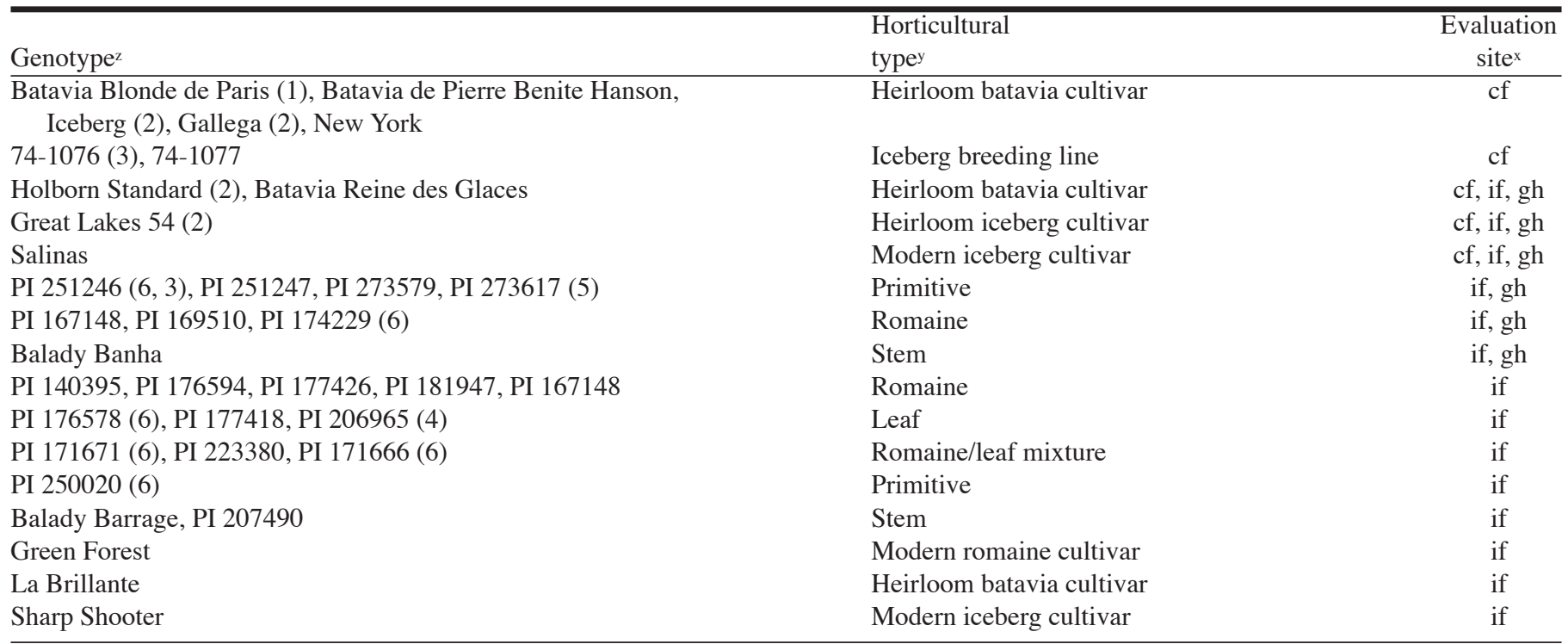

zNumbers in parentheses refer to the original report of resistance to $S$. sclerotiorum $(1,2,3,4)$ or $S$. minor (5, 6). References are 1: Chupp and Sherf, 1960; 2: Elia and Piglionica, 1964; 3: Whipps et al., 2002; 4: Newton and Sequeira, 1972; 5: Abawi et al., 1980 ; 6: Subbarao, 1998.

yLettuce types are as described by Ryder (1999). Genotypes with PI designations are accessions that are not commercially grown in the United States.

${ }^{\mathrm{x} f \mathrm{f}}=\mathrm{commercial}$ field, if $=$ inoculated field, and $\mathrm{gh}=$ greenhouse or a combination, as indicated. 


\begin{tabular}{|c|c|c|c|c|c|c|}
\hline Experiment & $\begin{array}{l}\text { No. of } \\
\text { replications }\end{array}$ & $\mathrm{EU}^{\mathrm{z}}$ & $\begin{array}{c}\text { Planting } \\
\text { date }\end{array}$ & $\begin{array}{c}\text { Inoculation } \\
\text { date }^{y}\end{array}$ & $\begin{array}{l}\text { Plant age at } \\
\text { final rating } \\
\text { (d) }\end{array}$ & $\begin{array}{l}\text { Inoculum } \\
\text { type }^{\mathrm{x}}\end{array}$ \\
\hline Field, 1980a & 2 & $62-115$ & $7-21-80$ & $\mathrm{n} / \mathrm{a}$ & 70 & Field \\
\hline Field, 2000 & 2 & $18-51$ & $8-17-00$ & $9-19-00$ & 74 & Field and rye \\
\hline Field, 2001 & 3 & $18-29$ & 8-9-01 & $9-26-01$ & 70 & Field and rye \\
\hline Greenhouse (GH) 1 & 4 & 9 & $3-31-00$ & $5-9-00$ & 80 & Rye \\
\hline $\mathrm{GH} 4$ & 3 & 12 & 4-17-02 & $5-22-02$ & 46 & Rye or PDA \\
\hline GH 5 & 3 & 12 & $5-23-02$ & $6-27-02$ & 46 & Rye or PDA \\
\hline GH 6 & 3 & 12 & $8-8-02$ & $9-12-02$ & 46 & Rye or PDA \\
\hline GH 7 & 3 & 12 & $9-12-02$ & $10-17-02$ & 46 & Rye or PDA \\
\hline
\end{tabular}

${ }^{\mathrm{z}}$ Experimental unit (EU) is the number of plants evaluated per genotype per replicate.

yn/a = no supplemental inoculum was added.

xPlants were exposed either to $S$. minor field populations (field), rye seed infested with S. minor (rye), PDA plugs infested with $S$. minor(PDA),

USDA-ARS research station in Salinas, Calif. High levels of $S$. minor inoculum were established at this site through a combination of continuous cropping with lettuce and the incorporation of rye seeds colonized by $S$. minor, prepared as described above, every 1 to 3 years for over a decade.

In 1980, each experimental unit comprised a $15.2 \mathrm{~m}$ double-row plot, and in 2000 and 2001, each experimental unit comprised a 7.6 $\mathrm{m}$ double-row plot. For all field experiments, lettuce seeds were sown directly in two rows on beds $102 \mathrm{~cm}$ wide and thinned after 4 weeks to $31 \mathrm{~cm}$ spacing. Additional experimental details are given in Table 2. In both 2000 and 2001, field populations of $S$. minor were supplemented by placing one or two rye seeds colonized with $S$. minor 1 to $2 \mathrm{~cm}$ from the base of each plant after thinning. Initial stand counts were taken immediately following inoculation. Plants were monitored for the appearance of lettuce drop symptoms and DI (proportion of dead plants) was determined weekly. Data from only the final evaluation date are presented.

Resistance EVAluATION-GREenhouse. Lettuce seeds were sown directly or transplanted 14 to $21 \mathrm{~d}$ after seeding into 7.5 $\mathrm{cm}$ pots containing Salinas Valley field soil treated with sodium methyldithiocarb. Experimental design details for each experiment are given in Table 2. Plants were inoculated at 5 weeks of age by pressing infested rye seed or PDA plug inoculum into the soil surface $\approx 1 \mathrm{~cm}$ from the base of the plant. Plants were watered carefully following inoculation to prevent movement of the inoculum. Plants were monitored daily for mycelial growth at the plant base and subsequent death due to lettuce drop for at least $19 \mathrm{~d}$ following inoculation.

Statistical Methods. For all experiments, treatments were randomly assigned to experimental units within replicates. Data were analyzed by analysis of variance (ANOVA) using the general linear model procedure of JMP v. 4.0.2 (SAS Institute, Cary, NC). Before ANOVA testing, all data were evaluated for normality and homogeneity of variances within and between experiments. DI values were subjected to the arcsin transformation for binomial proportions with Bartlett's correction for proportions of zero and one (Snedecor and Cochran, 1989). Genotypes were considered fixed effects, and replicate, experiment and year were considered random effects. Significant differences among genotypes were tested using the (genotype $\times$ experiment) interaction mean square as the denominator for the F test. For comparisons between genotypes within a single test, least significant differences were calculated using Tukey's or Dunnett's multiple comparisons pro- cedures, as specified, using an experimentwise error rate of $\alpha=$ 0.05 (Chew, 1977). Pearson product-moment correlations were calculated using the multivariate platform of JMP to compare results obtained in different resistance evaluation experiments. Throughout this report, the term disease rating (DR) refers to transformed DI values, calculated as follows: $D R=\arcsin (\sqrt{D I})$, where DI $=[$ (total number of dead plants $) /($ number of plants at start of experiment).

\section{Results}

Resistance EVALUATION-COMMERCIAL FIELDS. In all field experiments, infected plants initially displayed mild wilting and yellowing, followed by complete collapse, soft rot, and death. The interval between the initial observation of symptoms and plant death was usually $<1$ week. After plant collapse, abundant white mycelia and sclerotia characteristic of $S$. minor covered plant tissues.

The incidence of lettuce drop was moderate at both commercial field sites, with average DR of 28.7 in 1980a and 19.3 in 1980b (Table 3). Significant variation in susceptibility to $S$. minor was observed among the 12 genotypes evaluated $(P<0.001)$, with DR ranging from 19.8 to 58.8 in 1980 a and from 9.3 to 49.7 in 1980b. Different genotypes performed comparably in the two sites, as indicated by similar relative rankings and a strong correlation between observed DR ( $r=0.91 ; P<0.001)$. The control, 'Batavia Reine des Glaces' (BRG), was the most susceptible genotype at both sites, with an overall DR of 54.2. In comparison, the representative modern 'Salinas' exhibited DR of only 24.8. All of the genotypes tested had significantly lower DR than BRG, but only two, 'Great Lakes 54' ('GL54') and breeding line 74-1077, were significantly more resistant than 'Salinas' according to Tukey's test.

Resistance EVAluation-inOculated FiELD. Symptoms of lettuce drop were first apparent on a few plants one week after supplemental inoculum was applied, and DI increased steadily until termination of the experiment at crop maturity. Border rows of BRG surrounding the research plot uniformly exhibited a high incidence of disease.

The genotypes tested exhibited a wide range of incidence of lettuce drop (Table 4). None were immune, but most exhibited significantly lower DR than BRG. The 29 genotypes were of diverse geographical origin and varied growth habits, including primitive, stem, romaine and leaf lettuce types (Table 1). Those with the lowest DR were primitive L. sativa, stem or romaine lettuces with relatively 
Table 3. Lettuce drop disease ratings in two commercial field sites in the Salinas Valley of California, Blanco District, in 1980.

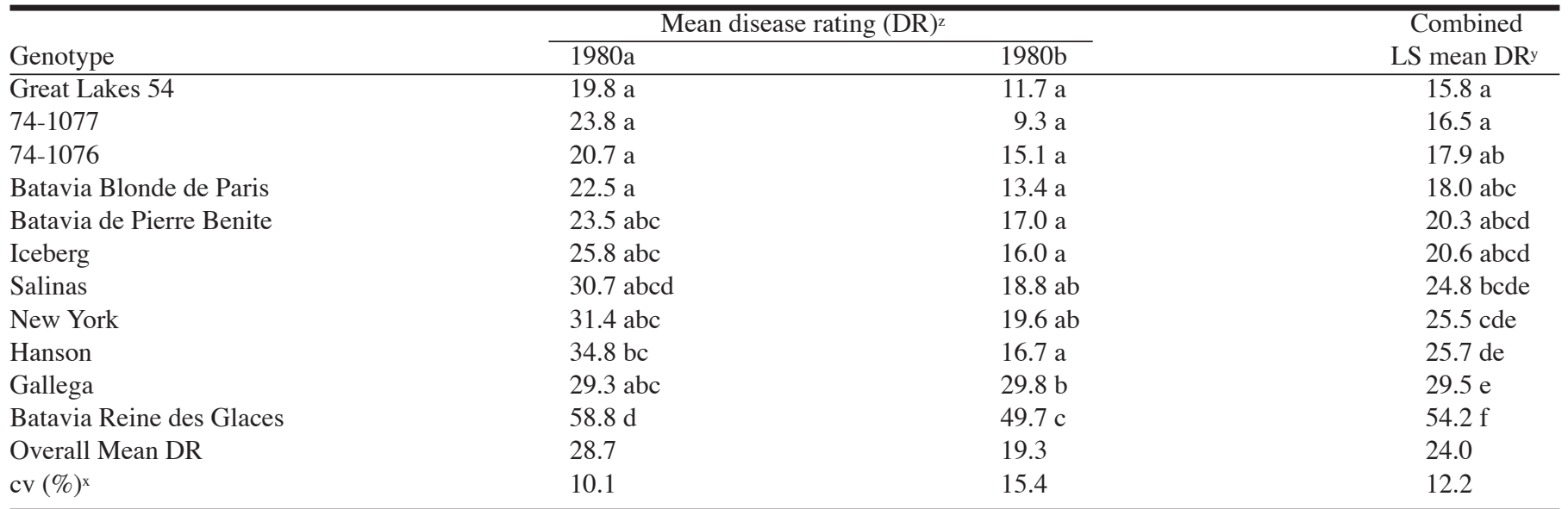

${ }^{2} \mathrm{DR}=$ arcsin-transformed disease incidence. Means within a column followed by different letters are significantly different $(P \leq 0.05)$ based on Tukey's test.

yLS mean = least squares mean from ANOVA of combined data from both tests.

${ }^{x}$ Coefficient of variation (CV), the square root of the mean squared error of ANOVA divided by the overall mean DR for the experiment.

Table 4. Reactions of 29 lettuce genotypes to lettuce drop in field inoculated with Sclerotinia minor in 2001.

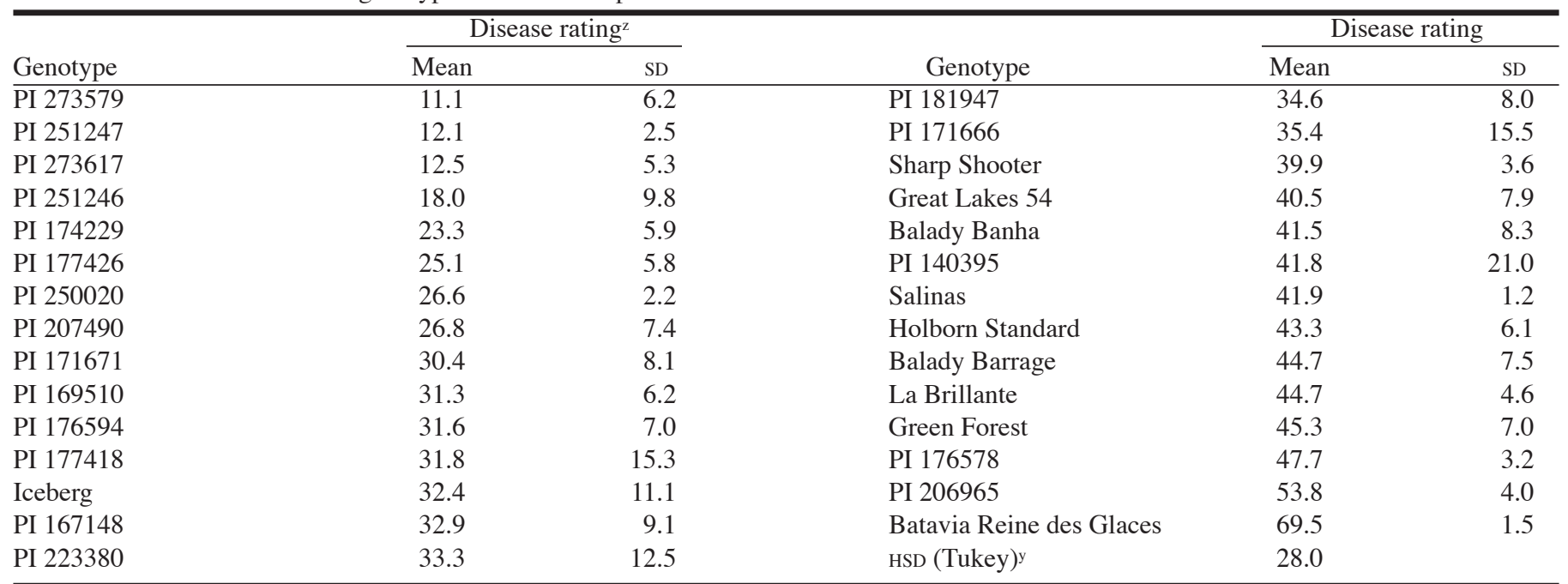

${ }^{2}$ Mean and SD of disease rating, arcsin-transformed disease incidence.

yThe least significant difference for Tukey's test for all pairwise comparisons at $P \leq 0.05$.

erect growth habits. Three of the genotypes, early-bolting primitive L. sativa accessions PI 273579, PI 251247 and PI 273617, had significantly lower DR than GL54, which was the most resistant cultivar in the commercial field tests. These also had significantly lower DR than the modern iceberg ('Sharp Shooter' and 'Salinas') and romaine ('Green Forest') cultivars. Neither 'Sharp Shooter' nor 'Green Forest', both described as tolerant to $S$. minor, were significantly more resistant than 'Salinas'.

CONSISTENCY BETWEEN FIELD EXPERIMENTS. To compare results obtained in different field experiments, 12 genotypes were evaluated in two consecutive years in the inoculated field (Table 5). In both experiments, significant variation in DR was observed among the 12 genotypes ( $P=0.002$ and $P<0.001$, respectively). The genotype $\mathrm{x}$ year interaction was not significant $(P=0.99)$, showing that genotypes performed similarly in both years. For example, the same four PI accessions had the lowest DR and BRG had the highest DR. The most notable discrepancy was for the cultivar Holborn Standard, which had the fifth lowest DR in 2000 but was one of the most susceptible genotypes in 2001. Despite this difference, overall disease ratings of the cultivars for the 2000 and 2001 field trials were strongly correlated $(r=0.92 ; P<0.001)$. Four of the genotypes were evaluated in commercial fields and inoculated field tests, allowing a direct comparison of the two environments. Although the susceptible control, BRG, exhibited DR of 67.3 and 69.5 in the inoculated field experiments, which was slightly higher than the DR observed in commercial fields, the disease ratings for the common genotypes were significantly correlated $(r=0.85 ; P<0.0001)$.

COMPARISON OF RESISTANCE EVALUATION METHODS. To determine whether results obtained in greenhouse tests are similar to those obtained in the field, the 12 genotypes evaluated in the 2000 and 2001 field experiments were inoculated in the greenhouse using two types of inocula. Infested rye seed was used as inoculum in seven tests, and in six of the seven, PDA plugs infested with mycelia were also used. In all experiments and using both procedures, symptoms of lettuce drop were first seen as early as 3 $\mathrm{d}$ after inoculation. Symptoms were similar to those seen in the field, and death typically occurred within $2 \mathrm{~d}$ after symptoms were first observed. The susceptible control was consistently infected and the overall mean DR was moderately high $(21.2$ 
Table 5. Lettuce drop disease ratings of 12 lettuce genotypes in response to inoculation with Sclerotinia minor in field and greenhouse (GH) experiments.

\begin{tabular}{lccc}
\hline & \multicolumn{3}{c}{ Mean disease rating (DR)z } \\
\cline { 2 - 4 } Genotype & Field, 2000 & Field, 2001 & Greenhouse, all combined \\
\hline PI 251246 & $19.0 \mathrm{a}$ & $18.0 \mathrm{abc}$ & $29.9 \mathrm{a}$ \\
PI 251247 & $21.3 \mathrm{a}$ & $12.1 \mathrm{a}$ & $37.9 \mathrm{abcd}$ \\
PI 273617 & $24.7 \mathrm{a}$ & $12.5 \mathrm{ab}$ & $32.4 \mathrm{ab}$ \\
PI 273579 & $25.2 \mathrm{a}$ & $11.1 \mathrm{a}$ & $33.1 \mathrm{abc}$ \\
Holborn Standard & $32.3 \mathrm{a}$ & $43.3 \mathrm{e}$ & $45.1 \mathrm{de}$ \\
PI 174229 & $32.4 \mathrm{a}$ & $24.0 \mathrm{abcd}$ & $39.6 \mathrm{bcd}$ \\
PI 169510 & $33.7 \mathrm{a}$ & $31.3 \mathrm{bcde}$ & $41.7 \mathrm{~d}$ \\
Balady Banha & $39.5 \mathrm{ab}$ & $41.5 \mathrm{de}$ & $39.7 \mathrm{bcd}$ \\
Great Lakes 54 & $39.8 \mathrm{ab}$ & $40.5 \mathrm{de}$ & $42.0 \mathrm{~d}$ \\
PI 167148 & $42.7 \mathrm{ab}$ & $32.9 \mathrm{cde}$ & $42.1 \mathrm{~d}$ \\
Salinas & $45.7 \mathrm{ab}$ & $41.9 \mathrm{de}$ & $40.7 \mathrm{~cd}$ \\
Batavia Reine des Glaces & $67.3 \mathrm{~b}$ & $69.5 \mathrm{f}$ & $52.4 \mathrm{e}$ \\
Overall Mean DR & 37.8 & 31.6 & 40.6 \\
CV $(\%)^{x}$ & 17.8 & 21.4 & 26.8 \\
\hline
\end{tabular}

${ }^{2} \mathrm{DR}=$ arcsin-transformed disease incidence. Means within a column followed by different letters are significantly different $(P \leq 0.05)$ based on

Tukey's test.

yLeast squares mean DR for each genotype from ANOVA of data from all greenhouse tests using both rye seed and PDA plug inocula.

${ }^{x}$ Coefficient of variation (CV), the square root of the mean squared error of ANOVA divided by the overall mean DR for the experiment.

Table 6. Analysis of variance of lettuce drop disease ratings for twelve genotypes in seven greenhouse experiments using $S$. minor-infested rye seeds or PDA plugs as inoculum.

\begin{tabular}{lcc}
\hline Source of variation & DF & F ratio \\
\hline GT & 11 & $9.9^{* *}$ \\
EXPT & 6 & $151.3^{* *}$ \\
GT $\times$ EXPT & 66 & 1.2 \\
Inoculum (EXPT) & 6 & $24.3^{* *}$ \\
Inoculum $\times$ GT $($ EXPT) & 66 & 1.0 \\
\hline
\end{tabular}

zAbbreviations: GT = genotype; EXPT = experiment.

to 67.2) in all tests, except when rye seed inoculum was used in test GH2 (9.0).

When data from all of the greenhouse tests were analyzed together, significant differences were observed between genotypes, between tests and between the two inoculation methods within test (Table 6). Genotype $\times$ test and genotype $\times$ inoculation method interactions were not significant, however, suggesting that genotypes performed similarly, relative to one another, in all experiments. The ANOVA least squared mean DR for the twelve genotypes analysed across tests were highly correlated with DR observed in field experiments $(r=0.86$ and 0.89 for 2000 and 2001 field tests, respectively) (Table 5). Accordingly, the most resistant (PI 251246, 251247, 273617, and 273579) and most susceptible (BRG) genotypes in greenhouse tests were the same as those identified in field tests (Table 5).

Although significant differences were detected between geno- types across all greenhouse tests, differences between genotypes were not always significant for individual experiments. Further, while the ranks of different genotypes were generally similar to those observed in field tests, there were some discrepancies. For example, genotypes that were among the most resistant in both field tests (e.g. PI 251247 and PI 273617) were among the most susceptible in some greenhouse tests (GH4, GH6, and GH7). The converse was also true; while 'Salinas' was one of the most susceptible genotypes in both field tests, it was among the most resistant in GH3 and GH5. As a result, although the results from some greenhouse tests were significantly correlated with the field tests, others were not (Table 7).

In the six tests where the two types of inoculum (rye seed, PDA plug) were compared, the two often did not give the same results. Although results using both types of inoculum were correlated with field tests in GH3 and GH5, only one or the other, but not both, were correlated with field results in GH4 and GH6. Finally, in $\mathrm{GH} 7$, neither method gave results that were correlated with field tests. Of the seven tests using rye seed inoculum, two were strongly correlated with field results, two were moderately correlated, and three showed no significant correlation. Similarly, for the six tests using PDA plug inoculum, three were strongly or moderately correlated with field results in both years, and three were not correlated with field results or were moderately correlated with field results in one year only. While neither type of inoculum appeared to be more consistently correlated with field results, BRG had the highest DR more consistently when PDA plugs were used.

Table 7. Correlations between lettuce drop disease ratings of twelve genotypes in field tests and in response to inoculation with Sclerotinia minor in greenhouse experiments using two types of inoculum.

\begin{tabular}{lcccccccc}
\hline & \multicolumn{9}{c}{ Greenhouse test } & \multicolumn{4}{c}{ Field } \\
\cline { 2 - 7 } Inoculum & GH 1 & GH 2 & GH 3 & GH 4 & GH 5 & GH 6 & GH 7 & test \\
\hline Rye & $0.75^{* * z}$ & 0.23 & $0.58^{*}$ & 0.14 & $0.66^{*}$ & $0.88^{* *}$ & 0.26 & 2000 \\
PDA plug & $0.87^{* *}$ & 0.05 & $0.59^{*}$ & 0.36 & $0.61^{*}$ & $0.92^{* *}$ & 0.42 & 2001 \\
& NT & $0.62^{*}$ & $0.64^{*}$ & $0.82^{* *}$ & $0.70^{*}$ & 0.48 & 0.53 & 2000 \\
& NT & 0.44 & $0.73^{* *}$ & $0.73^{* *}$ & $0.72^{* *}$ & 0.32 & 0.42 & 2001 \\
\hline
\end{tabular}

zPearson product-moment correlations were calculated from mean disease rating of the 12 genotypes used in all tests. Significant pairwise correlations between greenhouse and field tests are denoted * and ** for $P \leq 0.05$ or 0.01 , respectively. 


\section{Discussion}

To our knowledge, this is the first demonstration of S. minor resistance in lettuce in which resistance was evaluated in field experiments. The significant contribution of genotype to the observed variation in disease rating and the consistency of field test results in different years suggests that genetic components play a major role in determining resistance to $S$. minor. The variation in susceptibility observed among cultivars and the extreme susceptibility of the heirloom cultivar BRG also suggests that response to selection may have already occurred within cultivated germplasm for this trait. The number and inheritance of genes controlling partial resistance have not been determined for any of the genotypes mentioned.

In commercial field tests of head lettuce cultivars, the heirloom 'Great Lakes 54' ('GL54') and the breeding line 74-1076 had significantly lower disease ratings than the modern 'Salinas'. The DR of GL54 was only 16.0, as compared with 24.0 for 'Salinas'. A true difference of this magnitude would represent an economically significant reduction in crop losses, and could be a valuable addition to modern cultivars. In inoculated field tests, however, the DR for 'GL54' and two modern cultivars that have been described as partially resistant to Sclerotinia, 'Sharp Shooter' and 'Green Forest', were equivalent to that of 'Salinas'. It is possible that the sample sizes and replication within the inoculated field tests were insufficient to detect small differences in resistance levels. Alternatively, it is possible that higher pathogen populations or increased disease pressure were present in the inoculated field, and that the partial resistance observed in commercial fields was overwhelmed by supplemental inoculum. If this is the case, it would suggest that the incorporating this partial resistance in modern cultivars would provide minimal improvement when the disease pressure is high.

In fields inoculated with $S$. minor, three non-heading PI accessions had higher levels of resistance than any cultivar, including 'GL54', 'Green Forest' and 'Sharp Shooter'. Several others had significantly lower mean DR than the susceptible control BRG and were considered moderately resistant. Resistance reactions of most genotypes were described for the first time in this report, but six of the genotypes that were moderately or highly resistant in the experimental field were previously described as exhibiting low DI, slow-dying resistance, or both, to $S$. minor in greenhouse tests (Table 1). This suggests that delayed death following infection (slow-dying) and lower incidence of disease, as we have measured, may have the same underlying genetic and mechanistic basis. In this case, screening for slow-dying may effectively identify genotypes that also have low disease incidence in infested fields. Although death due to lettuce drop often occurs just before harvest and a delay between infection and death could potentially increase the percentage of harvestable plants, infected plants would still succumb to the disease after harvest and could act as the source of future infections in storage. Therefore, a decrease in the incidence of infection remains our primary objective for resistance breeding.

In a recent study, 'Holborn Standard' and PI 206965 were susceptible and PI 251246 and 74-1076 were resistant to $S$. sclerotiorum (Whipps et al., 2002), which is in agreement with our results using S. minor. In another report, however, PI 206965 was described as field-resistant to S. sclerotiorum (Newton and Sequiera, 1972), which may suggest that lettuce drop resistance is pathogen species-specific in some conditions. In peanut, the only other major crop affected by both pathogen species, genotypes with resistance to $S$. minor were also resistant to $S$. sclerotiorum (Cruikshank et al., 2002). Although this suggests that it will be possible to identify resistance genes that are broadly effective, further studies are required to determine whether the same genes confer resistance to both $S$. minor and $S$. sclerotiorum in lettuce.

An association between growth habit and resistance to $S$. sclerotiorum has been observed for lettuce (Newton and Sequiera, 1972; Whipps et al., 2002), however, in greenhouse tests with $S$. minor, no association between horticultural type and resistance was detected (Abawi et al., 1980; Subbarao, 1998). In peanut, both physiological and architectural resistance to $S$. minor have been described (Cruikshank et al., 2002). The mechanism of $S$. minor resistance that we have described is not known. Although different horticultural types were not evenly represented among the genotypes evaluated in our study, we found that those with the highest levels of $S$. minor resistance were primitive, stem, or romaine-type lettuces with upright growth habits, many of which were early-bolting. Intermediate levels of resistance were observed, however, for less erect and later bolting genotypes. Ideally, the highest levels of resistance may be transferred to heading types with selection. If high levels of resistance are due to avoidance, however, transfer into horticulturally acceptable genetic backgrounds may be difficult or impossible. For example, early bolting could facilitate avoidance of infection by $S$. minor, since the lignified stem and crown tissues of bolting plants may be more resistant to degradation by fungal enzymes than the softer tissues of plants in the rosette stage. In this case, physiological resistance, even if the overall level of resistance is lower, may be more useful for developing resistant cultivars.

When data from several greenhouse tests were combined, the strong correlation between greenhouse and field test results suggests that partial resistance in the field may be predicted using greenhouse assays. To further support this conclusion, greenhouse screens were successfully used by our group and by others (Abawi et al., 1980; Subbarao, 1998) to identify accessions that showed partial resistance in field tests. The results obtained in individual tests were not consistently correlated with field results, however, suggesting that field performance may not be accurately predicted by a single greenhouse test. The success of an individual experiment may be evaluated through the inclusion of control genotypes, such as the 12 described in this report, that are known to give a range of responses in field tests. Of the two inoculation techniques evaluated, susceptible controls consistently had higher DI when inoculated with PDA plugs, and rye seed inoculum failed to infect in one experiment. Although this suggests that inoculation with PDA plugs may give more consistent results across tests, there was no evidence that either method was more likely to give results that were correlated with field performance. Our results reinforced the necessity of field evaluation in the context of a breeding program, and established that further work is needed to identify and control the variability between greenhouse tests. In the meantime, with adequate replication and repetition, either or both of the greenhouse methods discussed may be used to permit more rapid progress than is possible through field testing alone. Further optimization of resistance evaluation procedures will not only speed breeding progress, but will also facilitate future studies of the mechanisms and genetic basis of resistance.

\section{Literature Cited}

Abawi, G.S., R.W. Robinson, A.C. Cobb, and J.W. Shail. 1980. Reaction of lettuce germplasm to artificial inoculation with Sclerotinia minor under greenhouse conditions. Plant Dis. 64:668-671.

Bardin, S.D. and H.C. Huang. 2001. Research on biology and control of 
Sclerotinia diseases in Canada. Can. J. Plant Pathol. 23:88-98.

Chew, V. 1977. Comparison among treatment means in an analysis of variance. USDA-ARS, Wash., D.C.

Chupp, C. and A.F. Sherf. 1960. Vegetable diseases and their control. Ronald Press, New York.

Cruikshank, A.W., M. Cooper, and M.J. Ryley. 2002. Peanut resistance to Sclerotinia minor and S. sclerotiorum. Austral. J. Agr. Res. 53: $1105-1110$.

Elia, M. and V. Piglionica. 1964. Preliminary observations on the resistance of some lettuce cultivars to "collar rot" caused by Sclerotinia spp. Phytop. Medit. 3:37-39.

Hao, J.J., K.V. Subbarao, and S.T. Koike. 2003. Effects of broccoli rotation on lettuce drop caused by Sclerotinia minor and on the population density of sclerotia in soil. Plant Dis. 87:159-166.

Hollowell, J.E., B.B. Shew, and M.A. Cubeta, M.A. 2003. Weed species as hosts of Sclerotinia minor in peanut fields. Plant Dis. 87:197-199.

Koike, S.T., R.F. Smith, L.E. Jackson, L.J. Wyland, J.I. Inman, and W.E. Chaney. 1996. Phacelia, Lana woollypod vetch, and Austrian winter pea: Three new cover crop hosts of Sclerotinia minor in California. Plant Dis. 76:101.

Madjid, A., S. Honma, and M.L. Lacy. 1983. A greenhouse method for screening lettuce for resistance to Sclerotinia sclerotiorum. Sci. Hort. 18:201-206.
Newton, H.C. and L. Sequeira. 1972. Possible sources of resistance in lettuce to Sclerotinia sclerotiorum. Plant Dis. Rpt. 56:875-878.

Ryder, E.J. 1999. Lettuce, endive and chicory. Crop production in horticulture series. vol. 7. CABI Publ., CAB Intl., New York.

Seminis Vegetable Seeds. 1999. Lactuca sativa cultivar exhibiting resistance to downy mildew and corky root rot, U.S. patent no. 5973232.

Snedecor, G.W. and W.G. Cochran. 1989. Statistical methods. Iowa State Univ. Press, Ames.

Subbarao, K.V., J.C. Hubbard, and K.F. Schulbach. 1997. Comparison of lettuce diseases and yield under subsurface drip and furrow irrigation. Phytopathology 87:877-883.

Subbarao, K.V., S.T. Koike, and J.C. Hubbard. 1996. Effects of deep plowing on the distribution and density of Sclerotinia minor sclerotia and lettuce drop incidence. Plant Dis. 80:28-33.

Subbarao, K.V. 1998. Progress towards integrated management of lettuce drop. Plant Dis. 82:1068-1078.

Subbarao, K.V. 2000. Epidemiology and control of lettuce drop caused by Sclerotinia species, p. 105-118. E. Kurtz (ed.). Annu. Rpt. Calif. Lettuce Res. Board, Salinas, Calif.

Whipps, J.M., S.P. Budge, S. McClement, and D.A.C. Pink. 2002. A glasshouse cropping method for screening lettuce lines for resistance to Sclerotinia sclerotiorum. Eur. J. Plant Pathol. 108:373-378. 